\title{
A MACH-UNIFORM ALGORITHM: COUPLED VERSUS SEGREGATED APPROACH
}

\author{
Krista Nerinckx*, Jan Vierendeels* and Erik Dick* \\ ${ }^{*}$ Ghent University, Department of Flow, Heat and Combustion Mechancis \\ Sint-Pietersnieuwstraat 41, B-9000 Ghent, Belgium \\ e-mail: Krista.Nerinckx@UGent.be \\ web page: http://www.floheacom.ugent.be/
}

Key words: Mach-uniform, segregated algorithm, coupled algorithm, pressure-correction, Fourier analysis, inviscid flow, heat transfer, gravity

\begin{abstract}
A Mach-uniform algorithm is an algorithm with a good convergence rate for any level of the Mach number. In this paper, the severe time step restriction for low speed flows is removed by treating the acoustic and diffusive terms implicitly. After identification of these terms in the conservative set, we end up with a semi-implicit system. The way to solve this system can be chosen. Three different solution techniques are presented: a fully coupled algorithm, the coupled pressure and temperature correction algorithm form [1], and a fully segregated pressure-correction algorithm. We analyse the convergence behavior of the considered algorithms in some typical flow problems. Moreover, a Fourier stability analysis is done for each of the algorithms. For inviscid flow, the fully segregated and the fully coupled algorithm need about as much time steps to reach steady state. Therefore, the more segregation is introduced, the cheaper the calculation can be done. In case of heat transfer, the fully segregated pressure-correction algorithm suffers from a diffusive time step limit. This is not the case for the semi-segregated coupled pressure and temperature correction algorithm. Finally, when the gravity terms play an important role, only the fully coupled algorithm can avoid an additional time step restriction.
\end{abstract}

\section{Introduction}

Historically, algorithms for CFD have been grouped into two classes: coupled density based methods and segregated pressure based methods. Both types of algorithms have been adapted to make them Mach-uniform, i.e. applicable for all speeds (see f.e. the references in [1]). In this paper, we present the idea that only a classification with regard to the solution technique (coupled versus segregated) is valuable: three different types of algorithms are constructed, each of them based on the same idea to reach Mach-uniformity, but applying a different solution technique. 


\section{Semi-implicit system}

Mach-uniform efficiency implies a good convergence rate for any level of the Mach number. In [1] we explain how Mach-uniformity can be reached by treating the acoustic and diffusive (friction, heat conduction) terms implicitly. The convective terms, on the other hand, can be treated explicitly. A finite volume method for the two-dimensional Navier-Stokes equations therefore results in the following semi-implicit system

$$
\begin{array}{r}
{\left[\rho_{i}^{(m+1)}-\rho_{i}^{[m]}\right] V_{i} / \Delta t^{I}+\sum \rho_{f}^{[m]} w_{f}^{(m+1)} d s_{f}=0,} \\
{\left[(\rho v)_{i}^{(m+1)}-(\rho v)_{i}^{[m]}\right] V_{i} / \Delta t^{I}+\sum(\rho v w)_{f}^{[m]} d s_{f}+\sum p_{f}^{(m+1)} d x_{f}=} \\
\sum\left(\vec{\tau} \cdot \overline{1}_{y} \cdot \bar{n}\right)_{f}^{(m+1)} d s_{f}-\rho_{i} g V_{i}, \text { similar for } u \\
{\left[(\rho E)_{i}^{(m+1)}-(\rho E)_{i}^{[m]}\right] V_{i} / \Delta t^{I}+\sum(\rho H)_{f}^{[m]} w_{f}^{(m+1)} d s_{f}=} \\
\sum(\kappa \nabla T \cdot \bar{n})_{f}^{(m+1)} d s_{f}+\sum(\vec{\tau} \cdot \bar{w})_{f}^{(m+1)} \cdot \bar{n} d s_{f}-\rho_{i} v_{i} g V_{i},
\end{array}
$$

with $\rho$ the density, $p$ the pressure, $T$ the temperature, $E$ the total energy, $H$ the total enthalpy, $\bar{g}$ the gravitational acceleration vector, $\vec{\tau}$ the viscous stress tensor and $\kappa$ the heat conduction coefficient. The summation runs over all faces $f$ of the control volume around node $i . d s_{f}$ is the length of the face $f$ and $w_{f}$ represents the projection of the velocity vector $\bar{w}=u \overline{1}_{x}+v \overline{1}_{y}$ on the outward normal $\bar{n}$ of the face $f$, i.e. $w d s=u d y+v d x$.

In the convective fluxes, the transported quantities $u, v, H$ are upwinded using a higher order method. Like in [2], we use for the mass flux and the pressure at a face a blending of the high speed AUSM+ flux and a low-speed central flux. A pressure dissipation term is added to the mass flux to prevent pressure-velocity decoupling at low Mach numbers.

A multistage time stepping with $k$ stages is used,

$$
Q^{[0]}=Q^{n} ; \ldots ; Q^{[m+1]}=Q^{[0]}+\alpha_{m+1} \Delta t Q_{t}^{[m]} ; \ldots ; Q^{n+1}=Q^{[k]} .
$$

$n$ and $n+1$ represent respectively the old and the new time level, while $[m]$ and $[m+1]$ represent stage levels. $Q$ is the state vector and $\Delta t=t^{n+1}-t^{n}$ the time step. The slope $Q_{t}=\frac{\partial Q}{\partial t}$ is determined as $Q_{t}^{[m]}=\Delta Q^{[m]} / \Delta t^{I}=\left(Q^{(m+1)}-Q^{[m]}\right) / \Delta t^{I} . \Delta t^{I}$ a chosen internal time step. The unknown state $Q^{(m+1)}$ is determined from the semi-implicit system, and then inserted into the multistage time stepping, $Q^{[m+1]}=Q^{[0]}+\alpha_{m+1} \omega \Delta Q^{[m]}$, with $\omega=\Delta t / \Delta t^{I}$. In the following we will use a Fourier stability analysis to analyse the algorithms. Notice that $\Delta t^{I}$ determines the shape of the Fourier symbol, while $\omega$ acts as a scaling factor for this curve.

\section{Solution technique: coupled versus segregated}

The way in which this semi-implicit system is solved for the updated values $(m+1)$ can now be chosen: three different solution techniques are considered, varying between a fully coupled and a fully segregated approach. In a fully coupled method, the semiimplicit $(4 \mathrm{Nx} 4 \mathrm{~N})$-system (1)-(3) is solved in a coupled way, in each step of the multistage 
time stepping. As a second solution technique, we consider the coupled pressure and temperature algorithm from [1]. This algorithm finds its place in between the fully coupled and the fully segregated approach. An explicit convective predictor step is followed by an acoustic / diffusive corrector step in which a $(2 \mathrm{Nx} 2 \mathrm{~N})$-system of correction equations has to be solved. The third algorithm is a fully segregated pressure-correction algorithm with pressure corrections determined from the energy equation. As explained in [1], this fully segregated algorithm can be applied for a perfect gas flow without heat transfer. Here, the dimension of the system to be solved in each multistage step is $(\mathrm{NxN})$ only.

\section{Behavior in some typical flow problems}

\subsection{Inviscid flow}

The invicid flow of a perfect gas is considered. First, we focus on low speed flow. In the stability analysis we consider a flow in the x-direction aligned with the grid, at $M=0.001$. We use the Van Leer- $\kappa$ scheme for the transported quantities (with $\kappa=1 / 3$ ), and the multistage time stepping with four stages and standard coefficients. The time step $\Delta t^{I}$ is calculated from a chosen convective CFL number, $\Delta t^{I}=c f l^{I} \Delta x / u$ and the scaling factor $\omega$ is chosen.

Figure 1 shows the Fourier symbol. For the fully coupled algorithm (COUP), $c f l^{I}=$ has to be decreased down to 0.5 to obtain a good shaped curve. Next, the curve can be scaled up with $\omega=2.7$ before it crosses the time stepping curve.

In the predictor-corrector approach, we consider three algorithms: a pressure-correction algorithm with pressure corrections form the energy equation $(\mathrm{PE})$, one with pressure corrections from the continuity equation (classical approach) (PC), and the coupled pressure and temperature correction algorithm (PT). Explicit convective terms are used. Apparently, the PE algorithm can use the highest global CFL number (i.e. $c f l^{I} * \omega$ ). For the PT algorithm the global CFL number is somewhat lower. For the PC algorithm the global CFL number is the lowest. This confirms that, for the case of a perfect gas without heat transfer, the corrections should by determined from the energy equation.

As a numerical test case, we take the inviscid flow past a bump in a channel, with an inlet Mach number of $10^{-5}$. Figure 2 compares the convergence plots. First, the maximum value for $c f l^{I}$ was determined to keep the computation stable with $\omega=1$. Next, this maximum value for $c f l^{I}$ was kept constant and $\omega$ was increased as much as possible. For the predictor-corrector algorithms, the PE algorithm converges the fastest. The PC algorithm needs the most time to converge, due to the use of the continuity equation to determine the pressure (see [1]). The PT algorithm has a convergence rate in between the $\mathrm{PE}$ and the $\mathrm{PC}$ algorithm. Indeed, due to the influence of the continuity equation in the corrector step, the convergence slows down compared to the PE algorithm. The PE algorithm has the same convergence behavior as the fully coupled algorithm. However, the cost to solve one time step is lower for the PE algorithm: only a (NxN)-system has to be solved per time step, opposed to a $(4 \mathrm{Nx} 4 \mathrm{~N})$-system for the fully coupled method. 


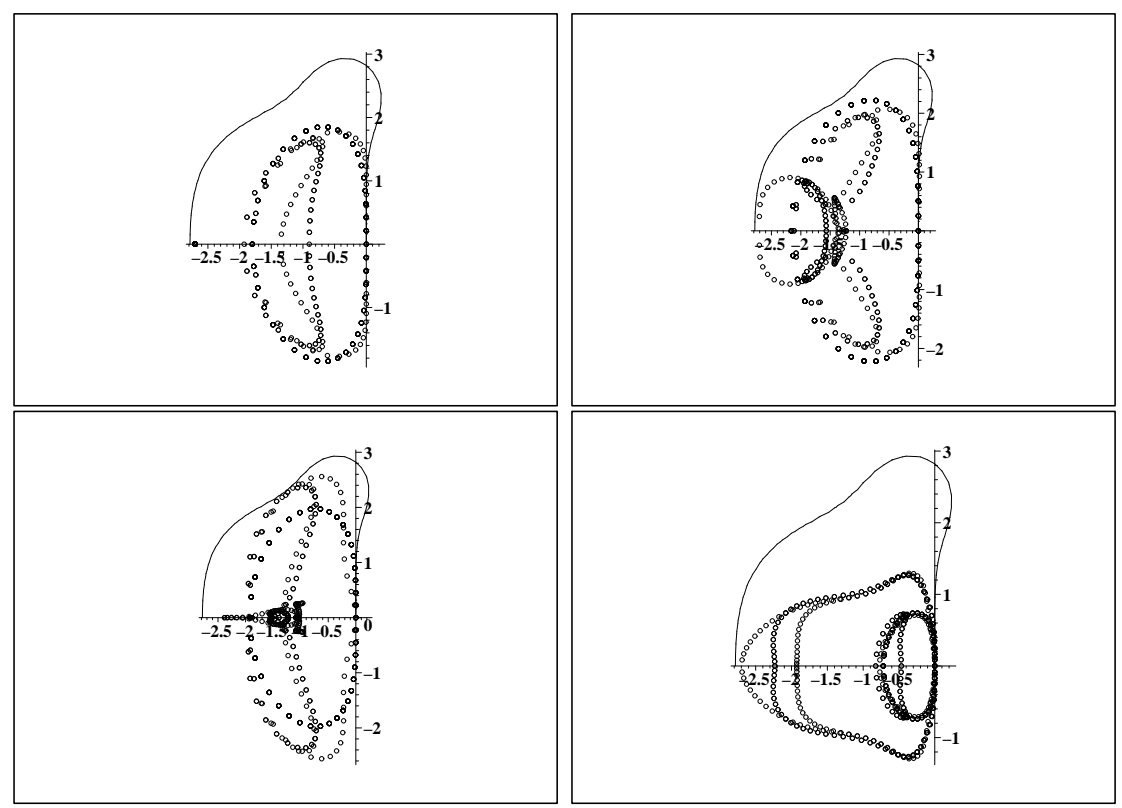

Figure 1: Fourier symbol, $M=0.001$. Top left: fully coupled algorithm, $c f l^{I}=0.5, \omega=2.7$. Top right: $\mathrm{PE}$ algorithm, $c f l^{I}=0.6, \omega=2.7$. Bottom left: PT algorithm, $c f l^{I}=0.6, \omega=2.4$. Bottom right: $\mathrm{PC}$ algorithm, $c f l^{I}=0.2, \omega=2.7$.

Therefore, the more segregation is introduced, the cheaper the simulation can be done.

Similar tests were done for high Mach number flow. The same conclusions hold.

\subsection{Heat transfer}

In [1] it was shown that the PE algorithm suffers from a diffusive time step limit due to the conductive terms. For the coupled pressure and temperature correction algorithm, however, such a limit is absent. The stability analysis now confirms this. The $c f l^{I}$ number is chosen as the maximum value for the Euler case and is kept constant. When the value of $\kappa$ is increased, the PE algorithm becomes unstable if $\kappa$ is taken too high. For the PT algorithm, however, $\kappa$ can be taken infinitely high.

Thus, the PT algorithm is advantageous for those cases where the heat conduction is important. What's more, for such a case the behavior of the PT algorithm improves. Indeed, in case of Euler equations, the convective CFL number can be taken at most 1.1 when implicit convective terms are used. However, if the diffusive terms dominate (i.e. a cell Peclet number $P e c<O(1)$ ), the convective CFL restriction is weakened, and even can disappear completely. This is confirmed by the Fourier stability analysis, where the CFL number can be taken higher as the value of Pec decreases. 


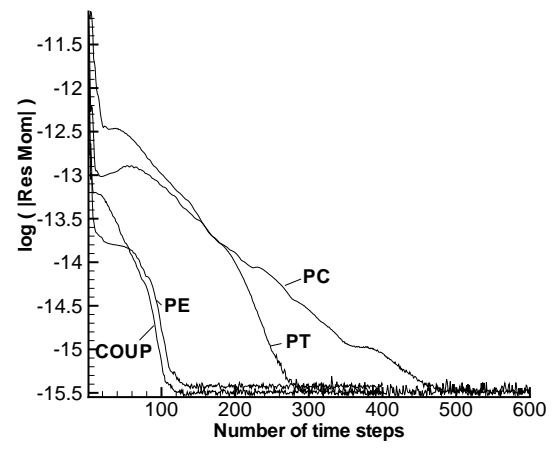

Figure 2: Test case bump, $M_{i n}=10^{-5}$. $\rho v$-momentum residual as a function of the number of time steps. COUP: $c f l^{I}=0.7, \omega=2.2$. PE: $c f l^{I}=0.7, \omega=2.9$. PT: $c f l^{I}=0.7, \omega=1.9$. PC: $c f l^{I}=0.5$, $\omega=1.8$.

\subsection{The role of gravity}

The gravity terms in the y-momentum and the energy equation act as source terms. Therefore, they may introduce an additional time step restriction. To analyse this, we add gravity terms to the Euler equations, and perform the Fourier stability analysis for a vertical flow at $M=0.001$.

In the fully coupled algorithm, the gravity terms can be treated implicitly without difficulties. For the Euler equations, i.e. $g=0$, the maximum value of the convective CFL number $c f l_{v}$ is 0.6 when a first order upwind discretisation is used for the transported quantities. We take $c f l_{v}=0.6$ and increase the value of $g$ (which represents some nondimensional number).

Figure 3 compares the eigenvalue plots for the algorithm without gravity $(g=0)$ and with an extremely high value for $g\left(g=10^{6}\right)$. We conclude that the fully coupled algorithm remains stable, no matter how high $g$ is taken. No additional time step restriction due to the gravity terms is introduced.

In a segregated predictor-corrector algorithm, however, it is no longer possible to treat the gravity terms in a fully implicit way. To get insight in the effect of the gravity term, we analyse the eigenvalues of the predictor amplification matrix. We find that the eigenvalues are completely determined by two nondimensional groups: the convective CFL number $c f l_{v}$ and a CFL number associated with the gravity force, $c f l_{g}=\frac{g \Delta t^{2}}{\Delta y}$. Next, we analyse the stability of the full predictor-corrector scheme as a function of the latter parameter, for the coupled pressure and temperature algorithm with implicit convective terms. Figure 3 shows the eigenvalue plots for $c f l_{v}=1$ and different values of $g$. For $g=10^{-7}\left(c f l_{g}=0.071\right)$ the eigenvalues are situated within the first order time stepping curve. For $g=10^{-5}\left(c f l_{g}=7.1\right)$, however, a part of the eigenvalue curve is situated in the right half plane. 


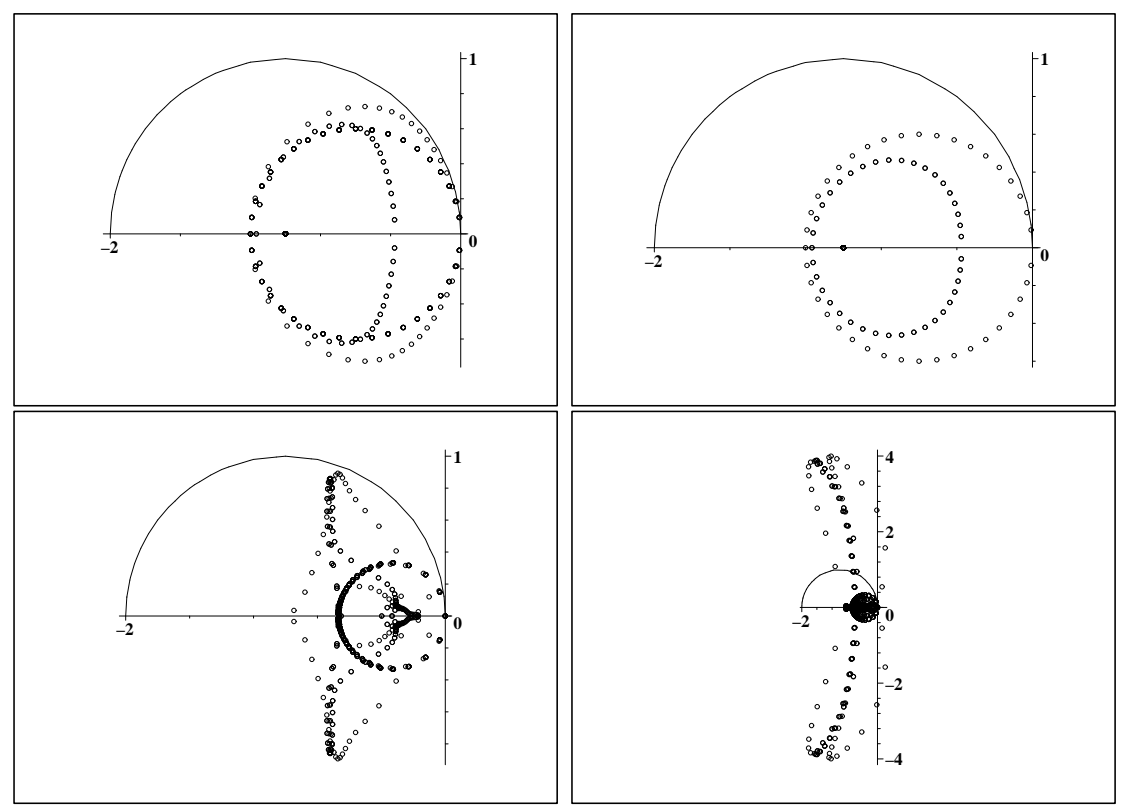

Figure 3: Fourier symbol. Top: fully coupled algorithm with implicit gravity terms, left: $g=0$, right: $g=10^{6}$. Bottom: PT algorithm, left: $g=10^{-7}, c f l_{g}=0.071$, right: $g=10^{-5}, c f l_{g}=7.1$.

Thus, for a segregated predictor-corrector algorithm, an additional time step restriction appears. This is a consequence of the segregation, i.e. the coupling between certain terms is broken up. This can be understood as follows. Consider the gravity term $-\rho g$ in the right hand side of the y-momentum equation. In a fully coupled algorithm, a perturbation of this term will provoke a response of the pressure: an equilibrium is created between the gravity force and the pressure gradient. In a segregated algorithm, however, the pressure is frozen in the predictor step, and therefore cannot respond directly to the gravity term. Consequently, a disturbance of the velocity field $v$ occurs. In the corrector step, the latter disturbance is transfered to the pressure field. In this way, the pressure responds to the gravity term in an indirect way. In order to prevent that the imbalance in the predictor step between pressure field and gravity force generates an excessively perturbed velocity field, the time step has to be chosen small enough. This explains the time step restriction due to gravity in a segregated algorithm.

\section{Conclusion}

We presented a Mach-uniform algorithm, using a semi-implicit approach. Three different solution techniques were considered to solve the semi-implicit system. Naturally, the fully coupled algorithm can do every flow problem without time step restrictions if all terms are treated implicitly. However, thinking of the computational cost to solve a large system, this is not beneficial. Indeed, the more segregation can be introduced, the cheaper the calculation can be done. Therefore, the right algorithm has to be used for 
the right application.

\section{Acknowledgments}

This research is funded with a fellowship granted by the Flemish Institute for the Promotion of Scientific and Technological Research in the Industry (IWT).

\section{REFERENCES}

[1] K. Nerinckx, J. Vierendeels, and E. Dick. Mach-uniformity through the coupled pressure and temperature correction algorithm. Journal of Computational Physics, 206:597-623, 2005.

[2] J. Vierendeels, B. Merci, and E. Dick. Blended AUSM+ method for all speeds and all grid aspect ratios. AIAA Journal, 39(12):2278-2282, 2001. 